\title{
Hybrid Beamforming with Switches and Phase Shifters over Frequency-Selective Channels
}

\author{
Sohail Payami, Mohsen Khalily, Tian Hong Loh, Konstantinos Nikitopoulos
}

\begin{abstract}
The recent studies on hybrid beamformers with a combination of switches and phase shifters indicate that such methods can reduce the cost and power consumption of massive multiple-input multiple-output (MIMO) systems. However, most of the works have focused on the scenarios with frequency-flat channel models. This letter proposes an effective approach for such systems in frequency-selective channels and presents the closedform expressions of the beamformer and the corresponding sumrates. Compared to the traditional subconnected structures, our approach with a significantly smaller number of phase shifters results in a promising performance.
\end{abstract}

Index Terms-Massive MIMO, hybrid beamforming, phase shifters, switches, frequency-selective channels.

\section{INTRODUCTION}

Fully-digital massive multiple-input multiple-output (MIMO) systems have tremendous advantages in providing high spectral efficiencies. However, equipping each antenna with a dedicated radio frequency $(\mathrm{RF})$ chain significantly increases the cost and power consumption of such systems. Hence, hybrid analog-anddigital beamformers have attracted a lot of attention as a means to provide a trade-off between spectral and energy efficiencies, [1]-[3]. In such systems, a small number of RF chains are connected to a large number of antennas via a network of phase shifters and/or switches.

Generally, switches consume less power compared to phase shifters, but they may also result in reduced array gains. In [4], phase shifter selection has been identified as an effective approach to provide a trade-off between energy and spectral efficiencies in hybrid beamformers with a fully-connected structure. In this technique, each phase shifter is equipped with an ON/OFF switch; and phase shifters that do not have a significant contribution to spectral efficiency are identified and turned off. This idea was then adapted for low-complexity and energy-efficient hybrid beamformers with a subconnected structure to reduce the number of phase shifters [5]-[7].

The existing literature has mainly focused on the design of hybrid beamformers with a reduced number of phase shifters over frequency-flat fading channel models, which is more suitable for narrow-band transmission [2], [8]. However, as the signal bandwidth increases, the number of resolvable multipath components in the channel also increases, which gives rise to frequency-selectivity in many practical applications. Although

S. Payami (email: sohail.payami@surrey.ac.uk), M. Khalily and K. Nikitopoulos are with Institute for Communication Systems (ICS), University of Surrey, UK. T. H. Loh is with the 5G \& Future Communications Technology Group, National Physical Laboratory (NPL), UK (email: tian.loh@npl.co.uk). We would like to acknowledge the support of University of Surrey 5GIC (http://www.surrey.ac.uk/5gic) for this work. there are some recent studies on designing hybrid beamformers that only consist of phase shifters [1], [9], [10], the existing work on hybrid beamformers with a combination of switches and phase shifters does not provide an understanding of the design procedures and performance evaluations in such scenarios. Besides, finding the beamforming weights for such systems becomes a challenging task as a difficult nonconvex optimization problem needs to be solved. With this motivation, this letter proposes an effective and low-complexity approach to derive closed-form expressions of the beamformer and analyze the achievable sum-rates for beamforming structures with a combination of switches and phase shifters over frequencyselective channels.

Notations: A and a represent a matrix and vector. $\mathbf{I}_{K}$ denotes $K$-dimensional identity matrix. $\mathbf{a}_{n}$ and $\mathbf{a}_{m,:}$ are the $n$-th column and $m$-th row of $\mathbf{A}$, respectively. $A_{m, n}$ and $\left|A_{m, n}\right|$ denote the $(m, n)$ element of $\mathbf{A}$ and its magnitude. $\mathbf{A}^{-1}, \operatorname{det}(\mathbf{A}), \mathbf{A}^{\mathrm{H}}$ and $\mathrm{E}[\mathbf{A}]$ denote inverse, determinant, Hermitian and expected value of $\mathbf{A}$, respectively. $\mathcal{C N}(\mathbf{a}, \mathbf{A})$ presents a random vector of complex Gaussian distributed elements with expected value a and covariance matrix A. $A(n)$ represents discrete-time sequence with $(n)$ denoting the discrete time index. Finally, $\tilde{A}(k)$ presents the discrete Fourier transform of $A(n)$ with $k$ denoting the discrete harmonics index of the frequency response.

\section{SYSTEM MODEL AND BACKGROUND}

Consider a single-cell multiuser massive MIMO uplink scenario in which a base station with $M$ omnidirectional antennas serves $U \ll M$ single-antenna users. At time index $n$, the transmit and receive signal vectors are denoted by $\mathbf{x}(n)$ and $\mathbf{y}(n)$, respectively In the following, a tapped delay line discretetime channel model with $D$ distinguishable multipath components (MPCs) in the delay domain is used; and the impulse response of the wireless channel at time index $n$ is $\mathbf{H}(n)=$ $\sum_{d=0}^{D-1} \mathbf{H}_{d} \delta(n-d)=\sum_{d=0}^{D-1} \mathbf{H}_{\mathrm{w}, d} \mathbf{D}_{d}^{1 / 2} \delta(n-d)$, where $\mathbf{H}_{d}, \mathbf{H}_{\mathrm{w}, d}$, and $\mathbf{D}_{d}^{1 / 2}$ denote the channel coefficients, small and large scale fading parameters of the $d$-th MPC, respectively. Without loss of generality, the entries of the diagonal matrix $\mathbf{D}_{d}^{1 / 2} \in \mathbb{R}^{U \times U}$ are modeled as $D_{d, u, u}=\exp \left(-\psi_{u} d\right) / \sum_{d^{\prime}=0}^{D-1} \exp \left(-\psi_{u} d^{\prime}\right)$, where $\psi_{u}=(u-1) / 5$ [9]. The elements of $\mathbf{H}_{\mathrm{w}, d} \in \mathbb{C}^{M \times U}$ contain the small scale fading coefficients of the $d$-th channel tap where $H_{\mathrm{w}, d, m, u} \sim \mathcal{C N}(0,1)$. The channel input-output relationship in the time domain are related as $\mathbf{y}(n)=\sum_{d=0}^{D-1} \mathbf{H}(d) \mathbf{x}(n-d)+\mathbf{z}(n)$, where $\mathbf{z} \in \mathbb{C}^{M \times 1}$ denotes the independent and identically distributed (i.i.d.) zero-mean additive white Gaussian noise vector with variance $\sigma^{2}$ and $\mathrm{E}\left[\mathbf{z}(n) \mathbf{z}^{\mathrm{H}}(n)\right]=\sigma^{2} \mathbf{I}_{M}$. 


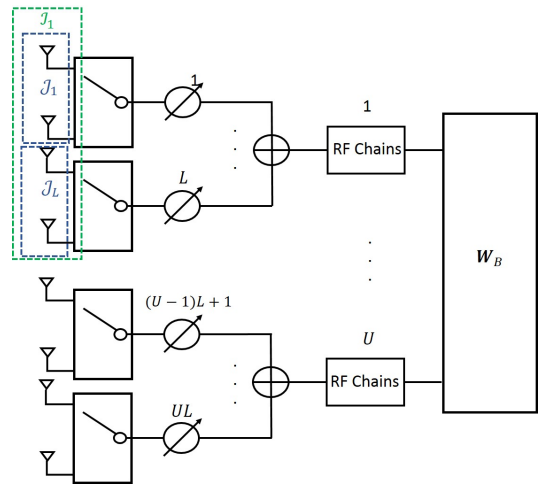

Fig. 1. Block diagram of the combiner at the base station with Subconnected structure with Reduced number of Phase Shifters (SRPS).

The base station applies a combining matrix with impulse response $\mathbf{W}(n) \in \mathbb{C}^{U \times M}$ to the received signal to reduce/eliminate the interference effects before sending the information to the detector. It is equipped with a hybrid beamformer with subconnected structure with reduced number of phase shifters, as shown in Fig. 1. To maximize the spatial multiplexing gain, we assume that the number of the RF chains is equal to the number of users $U$. The combining matrix $\mathbf{W}(n) \in \mathbb{C}^{U \times M}$ can be decomposed as $\mathbf{W}(n)=\mathbf{W}_{\mathrm{B}}(n) \mathbf{W}_{\mathrm{RF}}$ where $\mathbf{W}_{\mathrm{B}}(n) \in \mathbb{C}^{U \times U}$ and $\mathbf{W}_{\mathrm{RF}} \in \mathbb{C}^{U \times M}$ are the baseband combiner and RF beamforming matrices, respectively. Equivalently, in a system with $K$ subcarriers, the frequency response $\tilde{\mathbf{W}}(k)$ of the hybrid beamformer is expressed as $\tilde{\mathbf{W}}(k)=\tilde{\mathbf{W}}_{\mathrm{B}}(k) \tilde{\mathbf{W}}_{\mathrm{RF}}, \forall k \in\{0,1,2, \ldots, K-1\}$. In Fig. 1 each RF chain is connected to $L$ phase shifters, and each phase shifter can select one of the $S$ adjacent antennas. For the sake of clarity, let's label the RF chains, phase shifters and antennas in Fig. 1 from top to the bottom with integer indexes according to $u \in\{1, \ldots, U\}, i \in\{1, \ldots, U L\}, m \in\{1, \ldots, M\}$, respectively. Moreover, $\mathcal{J}_{i}=\{(i-1) S+1, \ldots, i S\}$ denotes a set that contains the index number of the antennas that are connected to the $i$-th phase shifter. The set $\mathcal{I}_{u}=\left\{\frac{M}{U}(u-1)+1, \ldots, \frac{M}{U} u\right\}$ includes the index number of the antennas that can be connected to the $u$-th $\mathrm{RF}$ chain. If the $m$-th antenna is connected to phase shifter $i$, where $m \in \mathcal{J}_{i}$ and $m \in \mathcal{I}_{u}$, then $W_{\mathrm{RF}, u, m}=e^{j \theta_{u, m}} / \sqrt{L}$ where $\theta_{u, m} \in[0,2 \pi)$. However, if the same antenna is not connected to a phase shifter, then $W_{\mathrm{RF}, u, m}=0$.

\section{iII. Problem Statement and the Proposed Solution}

For Gaussian entry inputs and additive white Gaussian noise, the uplink capacity of frequency-selective channel $\mathbf{H}(n)$ is

$$
\left.C(\mathbf{H})=\frac{1}{K} \sum_{k=0}^{K-1} \log _{2} \operatorname{det}\left(\mathbf{I}+\rho \tilde{\mathbf{H}}^{\mathrm{H}}(k) \tilde{\mathbf{H}}(k)\right)\right),
$$

where $\rho=P_{\mathrm{t}} / \sigma^{2}$ is a measure of signal-to-noise ratio (SNR) and $\tilde{\mathbf{H}}(k)$ is the frequency response of the channel and it is given by $\tilde{\mathbf{H}}(k)=\sum_{d=0}^{D-1} \mathbf{H}_{d} \exp \left(-\frac{j 2 \pi d k}{K}\right)$. In the existing literature on the design of hybrid beamformers over frequencyselective channels, the design criteria is [10]

$$
\left(\mathbf{W}_{\mathrm{B}}^{*}(k), \mathbf{W}_{\mathrm{RF}}^{*}\right)=\underset{\mathbf{W}_{\mathrm{B}}(k), \mathbf{W}_{\mathrm{RF}}}{\arg \max } R_{\mathrm{sum}}=\underset{\mathbf{W}_{\mathrm{B}}(k), \mathbf{W}_{\mathrm{RF}}}{\arg \max } \frac{1}{K} \sum_{k=1}^{K} R(k),
$$

where the sum-rate $R(k)$ over the $k$-th subcarrier is [10]

$$
\begin{aligned}
R(k)=\log _{2} \operatorname{det}\left(\mathbf{I}+\rho\left(\tilde{\mathbf{W}}_{\mathrm{B}}(k) \mathbf{W}_{\mathrm{RF}} \mathbf{W}_{\mathrm{RF}}^{\mathrm{H}} \tilde{\mathbf{W}}_{\mathrm{B}}^{\mathrm{H}}(k)\right)^{-1} \times\right. \\
\left.\tilde{\mathbf{W}}_{\mathrm{B}}(k) \mathbf{W}_{\mathrm{RF}} \tilde{\mathbf{H}}(k) \tilde{\mathbf{H}}^{\mathrm{H}}(k) \mathbf{W}_{\mathrm{RF}}^{\mathrm{H}} \tilde{\mathbf{W}}_{\mathrm{B}}^{\mathrm{H}}(k)\right) .
\end{aligned}
$$

This letter aims to design the beamforming weights $\left(\mathbf{W}_{\mathrm{B}}^{*}(k), \mathbf{W}_{\mathrm{RF}}^{*}\right)$ for hybrid beamforming systems with switches and phase shifters in frequency-selective channels such that the achievable sum-rate by the proposed scheme is maximized. This optimization is a challenging problem due to the nonconvex constraints $W_{\mathrm{RF}, u, m} \in\left\{0, e^{j \theta_{u, m}} / \sqrt{L}\right\}$ that are imposed by switches and phase shifters, respectively. To simplify the calculation of a low-complexity and near optimal beamformer for Fig. 1 and its performance, let's assume $M \rightarrow \infty$. As it will be shown in the simulation results section, the closedform expressions of sum-rates efficiency also provide a good approximation of performance for a more realistic $M$.

Relaxing the nonconvex constraints for the optimization in (2) is equivalent to assuming that the base station is equipped with a fully-digital system. In this case, linear methods such as zero-forcing (ZF) with $\tilde{\mathbf{W}}_{\mathrm{ZF}}(k)=\left(\tilde{\mathbf{H}}^{\mathrm{H}}(k) \tilde{\mathbf{H}}(k)\right)^{-1} \tilde{\mathbf{H}}^{\mathrm{H}}(k)$ and matched-filtering (MF) with $\tilde{\mathbf{W}}_{\mathrm{MF}}(k)=\tilde{\mathbf{H}}^{\mathrm{H}}(k)$ are nearoptimal solutions when $U \ll M$. ZF combiner can be viewed as a two-stage beamformer where the first-stage consists of a MF $\tilde{\mathbf{H}}^{\mathrm{H}}(k)$ and the second stage is a matrix inversion over the effective channel $\left(\tilde{\mathbf{H}}^{\mathrm{H}}(k) \tilde{\mathbf{H}}(k)\right)^{-1}$. With this background, hybrid beamforming with $\tilde{\mathbf{W}}(k)=\tilde{\mathbf{W}}_{\mathrm{B}}(k) \tilde{\mathbf{W}}_{\mathrm{RF}}$ can be viewed as a two-stage beamformer where $\tilde{\mathbf{W}}_{\mathrm{RF}}$ and $\tilde{\mathbf{W}}_{\mathrm{B}}(k)$ are the first and second stage beamformers respectively.

Instead of solving the optimization (2), we minimize the distance between the near-optimal ZF and our proposed beamformer. In other words, we design the RF beamformer according to minimum mean square error (MMSE) criteria

$$
\operatorname{minimize} \sum_{k=0}^{K-1}\left\|\tilde{\mathbf{W}}_{\mathrm{ZF}}(k)-\tilde{\mathbf{W}}_{\mathrm{B}}(k) \tilde{\mathbf{W}}_{\mathrm{RF}}\right\|^{2} / K \text {. }
$$

On the other hand, data processing equality indicates that the RF beamformer provides the performance bottleneck in hybrid beamforming systems and the baseband combiner is not be able to increase the achievable rates. More specifically, $R\left(\mathbf{H}_{\mathrm{e}}\right) \leq C\left(\mathbf{H}_{\mathrm{e}}\right) \leq C(\mathbf{H})$ where $C\left(\mathbf{H}_{\mathrm{e}}\right)$ and $R\left(\mathbf{H}_{\mathrm{e}}\right)$ denote the capacity of the effective channel $\mathbf{H}_{\mathrm{e}}(n)=\mathbf{W}_{\mathrm{RF}} \mathbf{H}(n)$ and the achievable sum-rate by the hybrid beamformer, respectively. Similar argument also holds for ZF combiner where the first stage beamformer is a MF, which can nearly preserve the capacity of the channel [9]. With this background, instead of solving (4), we design the RF beamformer according to

$$
\mathbf{W}_{\mathrm{RF}}^{*}=\underset{\mathbf{W}_{\mathrm{RF}}}{\arg \min } \sum_{k=0}^{K-1}\left\|\tilde{\mathbf{W}}_{\mathrm{MF}}(k)-\mathbf{W}_{\mathrm{RF}}\right\|^{2} / K .
$$

To solve (5), let's define $\tilde{\mathbf{E}}(k)=\tilde{\mathbf{W}}_{\mathrm{MF}}(k)-\mathbf{W}_{\mathrm{RF}}$. Then, Parseval's theorem states that the energy of a signal $\tilde{\mathbf{E}}$ in the frequency and time domains are equal. In other words,

$$
\frac{1}{K} \sum_{k=0}^{K-1}\|\tilde{\mathbf{E}}(k)\|^{2}=\sum_{n=0}^{K-1}\|\mathbf{E}(n)\|^{2} .
$$




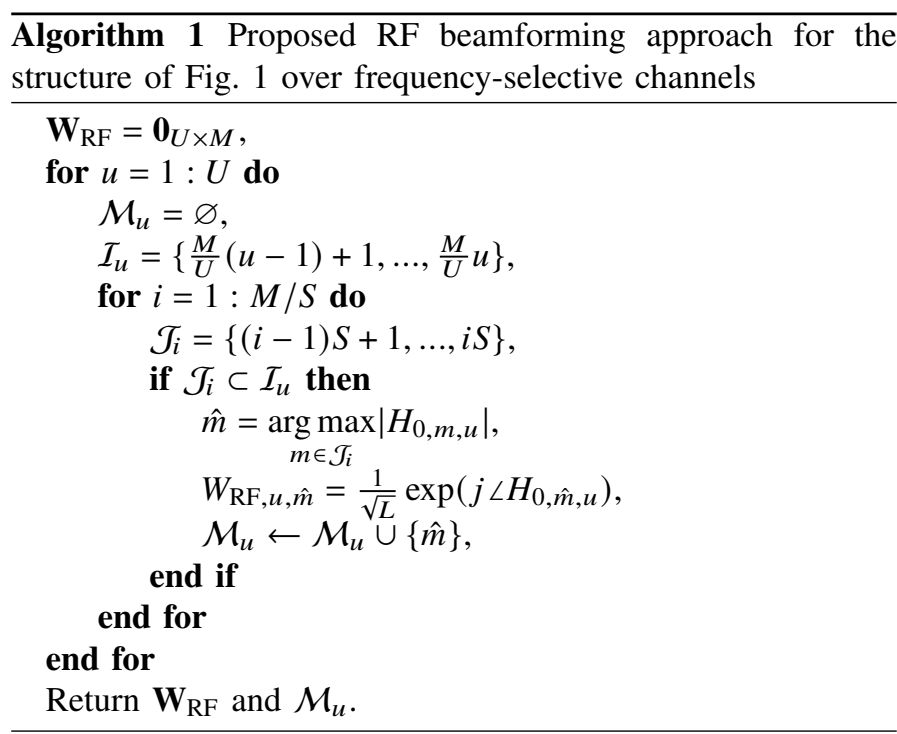

As the impulse response of the RF beamformer can be considered as a 1-tap filter, i.e., $\mathbf{W}_{\mathrm{RF}}(n \neq 0)=\mathbf{0}$, the optimal $\mathrm{RF}$ beamformer of (5) also minimizes

$$
\begin{gathered}
\left\|\mathbf{H}^{H}(0)-\mathbf{W}_{\mathrm{RF}}\right\|^{2}=\sum_{u=1}^{U} \sum_{m \in \mathcal{J}_{i}, i \in \mathcal{I}_{u}}\left|H_{u, m}^{\mathrm{H}}(0)-W_{\mathrm{RF}, u, m}\right|^{2} \\
+\sum_{u=1}^{U} \sum_{m \notin \mathcal{I}_{u}}^{M}\left|H_{u, m}^{H}(0)\right|^{2}+\sum_{u=1}^{U} \sum_{\substack{m \in \mathcal{J}_{i} \\
i \notin \mathcal{I}_{u}}}\left|H_{u, m}^{\mathrm{H}}(0)-W_{\mathrm{RF}, u, m}\right|^{2} .
\end{gathered}
$$

Since the last two terms are constant, (7) is minimized if the first term is minimized. It can be easily verified that the first term in (7) is minimized by setting

$$
W_{\mathrm{RF}, u, \hat{m}}^{*}=\frac{1}{\sqrt{L}} \exp \left(-j \angle H_{0, \hat{m}, u}\right),
$$

where $\hat{m}=\underset{m \in \mathcal{J}_{i}}{\arg \max }\left|H_{u, m}\right|$. Algorithm 1 summarizes the procedures to calculate $\mathbf{W}_{\mathrm{RF}}$ and $\mathcal{M}_{u}$, where $\mathcal{M}_{u}$ is a set that contains the indexes of the antennas that are connected to the $u$-th RF chain.

In the following, we analyze the impact of the proposed beamformer on the effective noise vector $\mathbf{z}_{\mathrm{e}}(n)=\mathbf{W}_{\mathrm{RF}} \mathbf{z}(n)$ and effective channel matrix which is defined as

$$
\tilde{\mathbf{H}}_{\mathrm{e}}(k)=\mathbf{H}_{\mathrm{e}, 0}+\sum_{d=1}^{D-1} \mathbf{H}_{\mathrm{e}, d} \exp \left(-\frac{j 2 \pi d k}{K}\right) .
$$

Due to the uncorrelated and circularly symmetric distribution of the elements of $\mathbf{H}_{\mathrm{w}}$ and $\mathbf{z}(n)$, it can be easily verified that $\mathrm{E}\left[\mathbf{W}_{\mathrm{RF}} \mathbf{z}(n) \mathbf{z}^{H}(n) \mathbf{W}_{\mathrm{RF}}^{H}\right]=\mathbf{W}_{\mathrm{RF}} E\left[\mathbf{z}(n) \mathbf{z}^{H}(n)\right] \mathbf{W}_{\mathrm{RF}}^{H}=$ $\sigma^{2} \mathbf{W}_{\mathrm{RF}} \mathbf{I} \mathbf{W}_{\mathrm{RF}}^{H} \rightarrow \sigma^{2} \mathbf{I}$. This indicates that the effective noise vector observed at the baseband is still additive white Gaussian after applying the proposed RF beamformer in (8). Similarly, the circularly symmetric property of the elements of $\mathbf{H}_{\mathrm{w}, d}$ results in $\forall d \neq 0, \frac{1}{\sqrt{L}} \mathbf{W}_{\mathrm{RF}} \mathbf{H}_{d} \rightarrow \mathbf{0}_{U \times U}$. Hence, the second term in (9) converges to zero; and this indicates that $\tilde{\mathbf{H}}_{\mathrm{e}}(k)=\mathbf{H}_{\mathrm{e}, 0}$, $\forall k \in\{0,1, \ldots, K-1\}$; and the effective channel by the proposed beamformer tends to have a flat frequency response when $M \rightarrow \infty$. To analyze $\mathbf{H}_{\mathrm{e}, 0}$, in the following we separately study the behaviors of its off-diagonal and diagonal elements of $\mathbf{W}_{\mathrm{RF}} \mathbf{H}_{\mathrm{w}, 0}$. The off-diagonal elements of $\mathbf{W}_{\mathrm{RF}} \mathbf{H}_{\mathrm{w}, 0}$ converge to zero as $\lim _{M \rightarrow \infty} \mathbf{w}_{\mathrm{RF}, u,:}: \mathbf{h}_{0, \mathrm{w}, u^{\prime}} \rightarrow 0, \forall u \neq u^{\prime}$ since the elements of $\mathbf{H}_{0, \mathrm{w}}$ are zero-mean, independent, circularly and symmetric. The diagonal elements of $\mathbf{W}_{\mathrm{RF}} \mathbf{H}_{\mathrm{w}, 0} \mathbf{D}_{0}$ are related to

$$
\begin{aligned}
\gamma & =\mathbf{w}_{\mathrm{RF}, u,:}, \mathbf{h}_{\mathrm{w}, 0, u}=\lim _{M \rightarrow \infty} \frac{1}{\sqrt{L}} \sum_{m \in \mathcal{M}_{m}}\left|H_{\mathrm{w}, 0, m, u}\right| \\
& =\lim _{M \rightarrow \infty} \frac{\sqrt{L}}{L} \sum_{m \in \mathcal{M}_{u}}\left|\sqrt{N} H_{\mathrm{w}, 0, m, u}\right| \stackrel{(a)}{=} \sqrt{L} \mathrm{E}[\breve{H}] \\
& \stackrel{(b)}{=} \sqrt{L} \sum_{s=0}^{S-1}\left(\begin{array}{c}
S-1 \\
s
\end{array}\right) \frac{(-1)^{s} S \sqrt{\pi}}{2(s+1)^{3 / 2}} .
\end{aligned}
$$

In (10), $(a)$ is directly inferred from product rule in calculating the limits, law of large numbers and the facts that the cardinality of $\mathcal{M}_{u}$ is $L$, and $(b)$ is deduced from $\mathrm{E}[\breve{H}]=$ $\sum_{s=0}^{S-1}\left(\begin{array}{c}S-1 \\ s\end{array}\right) \frac{(-1)^{s} S \sqrt{\pi}}{2(s+1)^{3 / 2}}, \forall S \geq 2$ [5], respectively. We define random variable $\breve{H}$ according to $\max _{m \in \mathcal{J}_{i}}\left|H_{\mathrm{w}, 0, m, u}\right|$. In other words, $\breve{H}$ is the maximum of $S$ Rayleigh distributed random variables $\left|H_{\mathrm{w}, 0, m, u}\right|$. Hence, the frequency response of the effective channel becomes

$$
\tilde{\mathbf{H}}_{\mathrm{e}}(k)=\gamma \mathbf{D}_{0}^{1 / 2}=\sum_{s=0}^{S-1}\left(\begin{array}{c}
S-1 \\
s
\end{array}\right) \frac{(-1)^{s} S \sqrt{\pi L}}{2(s+1)^{3 / 2}} \mathbf{D}_{0}^{1 / 2} .
$$

Inserting (11) into (1), the closed-form expressions of the sumcapacity capacity of the effective channel in the limit of $M \rightarrow$ $\infty$ (1) become

$$
C_{\mathrm{A}}\left(\mathbf{H}_{\mathrm{e}}\right)=\log _{2} \operatorname{det}\left|\mathbf{I}_{U}+\rho \gamma^{2} \mathbf{D}_{0}\right| .
$$

Remark 1. Traditional subconnected structures with one phase shifters per antenna, denoted by SP in the following, is equivalent to Fig. 1 with $S=1$ where all switches are always connected. For $S=1$, it can be easily shown that (11) becomes $\gamma=\mathbf{w}_{\mathrm{RF}, u,:} \mathbf{h}_{\mathrm{w}, 0, u}=\lim _{M \rightarrow \infty} \frac{1}{\sqrt{L}} \sum_{m \in \mathcal{I}_{u}}\left|H_{\mathrm{w}, 0, m, u}\right|=\sqrt{\pi L} / 2$. Consequently, the capacity of the effective channel can be calculated by inserting $\gamma=\sqrt{\pi L} / 2$ into (12).

The equation for the effective channel in (11) indicates that the interuser and intersymbol interference converge to zero as $M \rightarrow \infty$. However, the number of antennas in practical systems is limited, and hence, there may be residual interference in the system. As it will be shown by simulations in the next section, applying zero-forcing per subcarrier over the effective channel, at the baseband, results in a near-optimal performance as $R\left(\mathbf{H}_{\mathrm{e}}\right)$ by $\mathrm{ZF}$ is almost the same as $C\left(\mathbf{H}_{\mathrm{e}}\right)$. However, $\mathrm{ZF}$ combiner can be replaced with other methods such as minimum mean square error, depending on the design requirements.

Remark 2. The computational complexity of selecting the maximum of $S$ variables is related to $O(S)$, in the worst case. Since the phase of each of the $M / S$ phase shifters is calculated by finding the maximum of $S$ variables, the computational complexity of the proposed RF beamformer is 


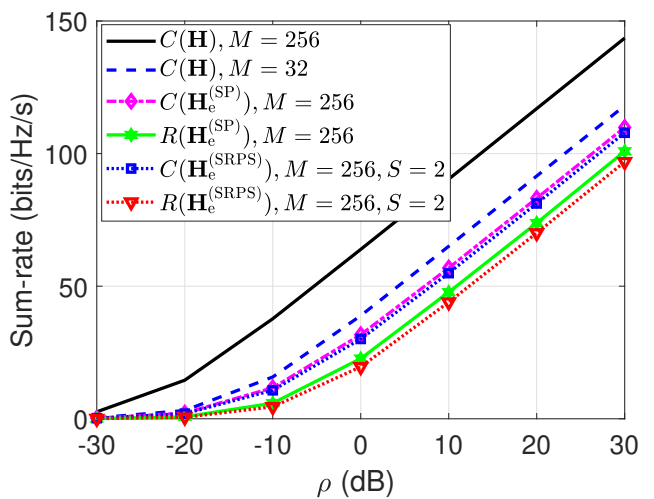

Fig. 2. Achievable sum-rates vs. $\rho$ for $M=256, U=8, S=2$.

related to $O(M / S . S)=O(M)$, in the worst case. As the signals travel from the antennas to the baseband, the matrix operations $\mathbf{W}_{\mathrm{RF}} \mathbf{y}(n)$ take place in the analog domain and impose no computations on the baseband. If the second-stage combiner is based on $\mathrm{ZF}$, with $O\left(K U^{3}\right)$, then the overall complexity of the proposed approach increases with $O\left(K U^{3}+M\right)$.

\section{Simulation Results}

In this section, Monte Carlo simulations averaged over 1000 realizations are used to evaluate the performance of the proposed method and the accuracy of the closed-form expressions, assuming $D=3$, and $K=64$. Let $C\left(\mathbf{H}_{\mathrm{e}}^{(\mathrm{SP})}\right), R\left(\mathbf{H}_{\mathrm{e}}^{(\mathrm{SP})}\right)$, $C\left(\mathbf{H}_{\mathrm{e}}^{(\mathrm{SRPS})}\right)$ and $R\left(\mathbf{H}_{\mathrm{e}}^{(\mathrm{SRPS})}\right)$ denote the sum-capacity and the achievable sum-rates by SP and SRPS structures, respectively. Fig. 2 presents the achievable sum-rates as a function of $\rho$ for $M=256, U=8$ and $S=2$. Fig. 2 also presents the capacity of $32 \times 8$ dimensional channel as the performance upper-bound as the array gain of subconnected structures is related to $M / U=32$. It should be noted that although fullydigital systems provide better sum-rates compared to hybrid structures, however, the corresponding energy efficiency of such systems is much lower when $U \ll M$ [6], [11]. Fig. 2 shows that $C\left(\mathbf{H}_{\mathrm{e}}^{(\mathrm{SRPS})}\right)$ by SRPS structure with 128 phase shifters, and $R\left(\mathbf{H}_{\mathrm{e}}^{(\mathrm{SP})}\right)$ with 256 phase shifters show comparable performance. Moreover, small values of $C\left(\mathbf{H}_{\mathrm{e}}^{(\mathrm{SP})}\right)-R\left(\mathbf{H}_{\mathrm{e}}^{(\mathrm{SP})}\right)$ and $C\left(\mathbf{H}_{\mathrm{e}}^{(\mathrm{SRPS})}\right)-R\left(\mathbf{H}_{\mathrm{e}}^{(\mathrm{SRPS})}\right)$ indicate that $\mathrm{ZF}$ can nearly reach the capacity of the effective channels. Let $C_{\mathrm{A}}\left(\mathbf{H}_{\mathrm{e}}^{\text {(SRPS) }}\right)$ and $C_{\mathrm{A}}\left(\mathbf{H}_{\mathrm{e}}^{(\mathrm{SP})}\right)$ denote the closed-form expressions of sum-capacity, by SRPS and SP structures, based on equations (11), (12) and Remark 1, respectively. For $M=512, U=8$ and different values of total number of phase shifters, given by $M / S$, Fig. 3 shows that the closed-form expressions provide an accurate approximation of the performance. In addition, the proposed approach for the SRPS with 64 phase shifters, equivalent to $S=8$ and only $12.5 \%$ of the phase shifters of the SP structure, results in $C_{\mathrm{A}}\left(\mathbf{H}_{\mathrm{e}}^{(\mathrm{SRPS})}\right) / C_{\mathrm{A}}\left(\mathbf{H}_{\mathrm{e}}^{(\mathrm{SP})}\right) \approx 84 \%$. Fig. 3 indicates that as the number of the phase shifters reduces, i.e., $S$ increases, using $\mathrm{ZF}$ results in higher performance loss compared to $C\left(\mathbf{H}_{\mathrm{e}}^{\text {(SRPS) }}\right)$. This indicates that other baseband combining techniques may be required for larger values of $S$.

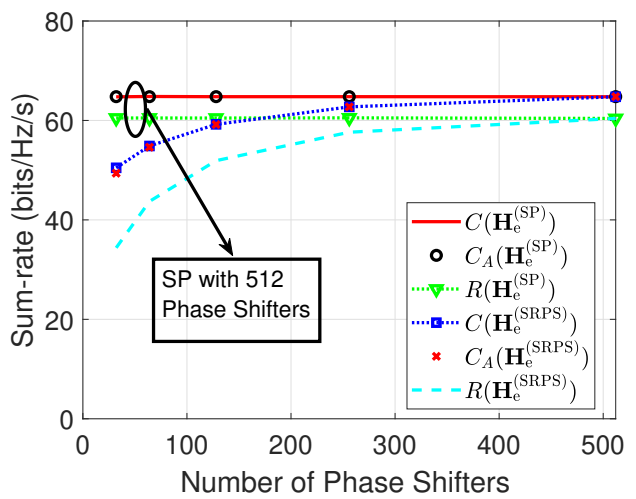

Fig. 3. Achievable sum-rates vs. the total number of phase shifters $M / S$, $M=512, U=8, S \in\{1,2,, 4, . ., 16\}, \rho=10 \mathrm{~dB}$.

\section{CONCLUSIONS}

This letter presented an effective hybrid beamforming approach for subconnected structures with a reduced number of phase shifters over frequency-selective channels. The closedform approximations of the proposed ZF-based beamformer and its performance can be used as a design guide to evaluate the performance of such structures. The proposed approach with a smaller number of phase shifters reaches a promising performance compared to the traditional subconnected structures.

\section{REFERENCES}

[1] I. Ahmed, H. Khammari, A. Shahid, A. Musa, K. S. Kim, E. De Poorter, and I. Moerman, "A survey on hybrid beamforming techniques in $5 \mathrm{G}$ : Architecture and system model perspectives," IEEE Communications Surveys Tutorials, vol. 20, no. 4, pp. 3060-3097, Fourthquarter 2018.

[2] S. S. Ioushua and Y. C. Eldar, "A family of hybrid analog-digital beamforming methods for massive MIMO systems," IEEE Transactions on Signal Processing, vol. 67, no. 12, pp. 3243-3257, June 2019.

[3] M. M. Molu, P. Xiao, M. Khalily, K. Cumanan, L. Zhang, and R. Tafazolli, "Low-complexity and robust hybrid beamforming design for multiantenna communication systems," IEEE Transactions on Wireless Communications, vol. 17, no. 3, pp. 1445-1459, March 2018.

[4] S. Payami, M. Ghoraishi, and M. Dianati, "Hybrid beamforming for large antenna arrays with phase shifter selection," IEEE Transactions on Wireless Communications, vol. 15, no. 11, pp. 7258-7271, Nov 2016.

[5] S. Payami, M. Ghoraishi, M. Dianati, and M. Sellathurai, "Hybrid beamforming with a reduced number of phase shifters for massive MIMO systems," IEEE Transactions on Vehicular Technology, vol. 67, no. 6, pp. 4843-4851, June 2018.

[6] S. Payami, N. Mysore Balasubramanya, C. Masouros, and M. Sellathurai, "Phase shifters versus switches: An energy efficiency perspective on hybrid beamforming," IEEE Wireless Communications Letters, vol. 8, no. 1, pp. 13-16, Feb 2019.

[7] S. Payami, C. Masouro, and M. Sellathurai, "Low-complexity and robust quantized hybrid beamforming and channel estimation," in 2018 IEEE Global Communications Conference (GLOBECOM), 2018, pp. 1-6.

[8] J. Eisenbeis, Y. Li, P. R. López, J. Fischer, and T. Zwick, "Comparison of hybrid beamforming systems using phase shifters and switches," in German Microwave Conference (GeMiC), March 2019, pp. 40-43.

[9] S. Payami, M. Sellathurai, and K. Nikitopoulos, "Low-complexity hybrid beamforming for massive MIMO systems in frequency-selective channels," IEEE Access, vol. 7, pp. 36 195-36 206, 2019.

[10] F. Sohrabi and W. Yu, "Hybrid analog and digital beamforming for mmWave OFDM large-scale antenna arrays," IEEE Journal on Selected Areas in Communications, vol. 35, no. 7, pp. 1432-1443, July 2017.

[11] C. G. Tsinos, S. Maleki, S. Chatzinotas, and B. Ottersten, "On the energyefficiency of hybrid analog-digital transceivers for single- and multicarrier large antenna array systems," IEEE Journal on Selected Areas in Communications, vol. 35, no. 9, pp. 1980-1995, Sept 2017. 